\title{
Cytogenetic analysis of adult T-Cell leukemia/ lymphoma: evaluation of a Caribbean cohort
} Yi Sun, Vundavalli V. Murty, Rebecca Leeman-Neill, Craig Soderquist, David
Park, Daniel B. Neill, Govind Bhagat \& Bachir Alobeid

To cite this article: Yi Sun, Vundavalli V. Murty, Rebecca Leeman-Neill, Craig Soderquist, David Park, Daniel B. Neill, Govind Bhagat \& Bachir Alobeid (2019) Cytogenetic analysis of adult T-Cell leukemia/lymphoma: evaluation of a Caribbean cohort, Leukemia \& Lymphoma, 60:6, 1598-1600, DOI: $10.1080 / 10428194.2018 .1538506$

To link to this article: https://doi.org/10.1080/10428194.2018.1538506

View supplementary material

央 Published online: 27 Nov 2018.

Submit your article to this journal

山 Article views: 95

View Crossmark data $\asymp$ 


\title{
Cytogenetic analysis of adult T-Cell leukemia/lymphoma: evaluation of a Caribbean cohort
}

\author{
Yi Sun ${ }^{a}$, Vundavalli V. Murty ${ }^{a}$, Rebecca Leeman-Neill ${ }^{a}$, Craig Soderquist ${ }^{a}$, David Park ${ }^{a}$, Daniel B. Neill ${ }^{b}$, \\ Govind Bhagat ${ }^{\mathrm{a}}$ and Bachir Alobeid ${ }^{\mathrm{a}}$
}

${ }^{a}$ Department of Pathology and Cell Biology, Columbia University Irving Medical Centre, New York Presbyterian Hospital, New York, NY, USA; ${ }^{b}$ Center for Urban Science and Progress, New York University, New York, NY, USA

ARTICLE HISTORY Received 7 July 2018; revised 7 October 2018; accepted 15 October 2018

Adult T-cell leukemia/lymphoma (ATLL) is a rare and highly aggressive type of peripheral T-cell lymphoma associated with human T-lymphotropic virus, type I (HTLV-I) infection. ATLL has a long latency and only a small percentage of HTLV-I infected individuals develop ATLL, suggesting the requirement of additional genetic events for neoplastic transformation of HTLV-I infected lymphocytes. The disease is endemic in several regions of the world, in particular, southwestern Japan and the Caribbean basin. The clinical features of Caribbean ATLL have been reported to differ from Japanese cases, including a younger age at diagnosis, higher frequency of the lymphomatous subtype, and a more aggressive clinical course [1-7]. A number of publications have described the cytogenetic profile of Japanese ATLL [8-11]; however, cytogenetic data on Caribbean ATLL are limited [5,6]. In this study, we assessed the cytogenetic alterations in a large series of Caribbean ATLL cases to investigate whether the differences in the types and frequencies of karyotype abnormalities account for the reported differences in clinical presentation and outcome between Japanese and Caribbean patients.

A total of 41 Caribbean ATLL patients diagnosed at our institution from 2003 to 2018 were evaluated. Clinical data, morphologic, immunophenotypic, and cytogenetic findings were reviewed. G-band karyotyping was performed after overnight and $72 \mathrm{hr}$ Phytohaemagglutininstimulated cultures and karyotypes described according to the International System for Human Cytogenomic Nomenclature (ISCN). Karyotype abnormalities were categorized as simple ( $<3$ aberrations) or complex ( $\geq 3$ aberrations). Each gain, loss or breakpoint is regarded as one aberration. Complex karyotypes were further sub-categorized as low complex (3-10 aberrations) and high complex (>10 aberrations). A total of 90 ATLL samples were analyzed (37 peripheral blood, 40 bone marrow, 7 lymph node, 1 subcutaneous mass, 1 mesenteric mass, 2 lung tissue, 1 skin biopsy, and 1 bronchial fluid) from the 41
Caribbean patients (26 F/15 M, age 16-73 years, median 52). The cases included 26 acute, 12 lymphomatous and 3 smoldering variants of ATLL. Abnormal karyotypes were detected in 45 samples from 31 patients. Thirty-four samples, including all 3 smoldering ATLL cases, demonstrated normal karyotypes and karyotyping failed in 11 samples. The tumors of 31 patients with abnormal karyotypes (in at least one sample) comprised acute $(n=22)$ and lymphomatous $(n=9)$ variants of ATLL were included in this analysis. Most patients received multiple chemotherapies, two patients also received stem cell transplant (Supplemental Table 1). The overall survival (OS-defined as the duration from initial diagnosis to the date of death, or the last follow-up) of these patients ranged from 10 days to 43 months (median 8 months) after initial diagnosis. The clinical data, cytogenetic findings, overall survival, and treatment information are summarized in Supplemental Table 1. Two-sided Mann-Whitney $U$ Test was used to compare the differences in overall survival and prognostic indices in each cytogenetic group (with and without particular cytogenetic abnormality).

All 31 patients exhibited complex karyotypes in at least one sample from each case. Low complex karyotypes were seen in 12 (38.7\%) and high complex karyotypes in $19(61.3 \%)$ cases. Near-diploid karyotype was seen in the majority of the patients $(n=24,77.4 \%)$ and near-tetraploid karyotypes in 7 patients (22.6\%). Karyotype heterogeneity (presence of subclones, defined as related clones with additional abnormalities compared to the stemline) was observed in 20 patients (64.5\%). Copy number losses most frequently involved chromosomes 14 (9 cases, 29.0\%), followed by 13 and 19 (7 each, 22.6\%), and 5 and 17 (6 each, 19.4\%). No consistent gain of chromosomes was seen. Among deletions, $6 q$ $(9,29.0 \%)$ and $3 q(7,22.6 \%)$ were most frequent. Recurrent rearrangement breakpoints were seen in more than $10 \%$ of patients at $1 q 21(6,19 \%), 3 p 21(6,19 \%)$, $6 q 21(8,26 \%), 9 p 13$ (4, 13\%), 14p11.2 (5, 16\%), 14q32

CONTACT Yi Sun sunyi969@gmail.com

(4) Supplemental data for this article can be accessed here.

(c) 2018 Informa UK Limited, trading as Taylor \& Francis Group 
Table 1. Major karyotype abnormalities and their correlations with survival in 31 Caribbean ATLL patients.

\begin{tabular}{|c|c|c|c|}
\hline Type of karyotype abnormality & $n(\%)$ & $\begin{array}{c}\text { Median } \\
\text { survival } \\
\text { (months) }\end{array}$ & $\begin{array}{c}p \text { value } \\
\text { (significance) }\end{array}$ \\
\hline Low complex (3-10 aberrations) & $12(38.7)$ & 12 & \\
\hline High complex (>10 aberrations) & $19(61.3)$ & 6.5 & .013 \\
\hline Hetrogeneity (with subclones) & $20(64.5)$ & 8 & .245 \\
\hline Near diploid & $24(77.4)$ & 8 & \\
\hline Near tetraploid & 7 (22.6) & 8 & .811 \\
\hline Chromosome 14 loss & $9(29.0)$ & 7 & .363 \\
\hline Chromosome 13 loss & $7(22.6)$ & 3 & $.043^{*}$ \\
\hline Chromosome 19 loss & $7(22.6)$ & & \\
\hline Chromosome 17 loss & $6(19.4)$ & & \\
\hline Chromosome 5 loss & $6(19.4)$ & & \\
\hline $\operatorname{Del}(6 q)$ & $9(29.0)$ & 7 & .384 \\
\hline Del $(3 q)$ & 7 (22.6) & & \\
\hline Del (17p) (including 17 loss) & $11(35.4)$ & 6.5 & .303 \\
\hline Marker \& HSR chromosomes & $20(64.5)$ & 8 & .531 \\
\hline \multicolumn{4}{|l|}{ Rearrangement breakpoints } \\
\hline $6 \mathrm{q} 21$ & $8(25.8)$ & & \\
\hline $1 \mathrm{q} 21$ & $6(19.4)$ & & \\
\hline $3 p 21$ & $6(19.4)$ & & \\
\hline $14 p 11.2$ & $5(16.1)$ & & \\
\hline $14 q 32$ & $5(16.1)$ & & \\
\hline $9 p 13$ & $4(12.9)$ & & \\
\hline $17 p 11.2$ & $4(12.9)$ & & \\
\hline $19 \mathrm{p} 13$ & $4(12.9)$ & & \\
\hline $19 q 13.3$ & $4(12.9)$ & & \\
\hline
\end{tabular}

All $p$ values were calculated in comparison to cases without the corresponding cytogenetic abnormalities.

${ }^{*} p$ value was calculated including two additional cases of del $13 q$.

$(5,16 \%), 17 p 11.2(4,13 \%), 19 p 13(4,13 \%)$, and 19q13.3 (4, 13\%) (Table 1). Additionally, homogenously staining regions, changes suggestive of gene amplification, were identified in two patients.

To assess any impact of cytogenetic abnormalities on clinical outcomes, we compared particular type or pattern of chromosomal changes with OS. We found high complex karyotype ( $>10$ abnormalities) was strongly associated with shorter survival (Table $1, p=.013$ ). The incidence of high complex karyotypes was more frequent $(12 / 16$ cases; $75 \%)$ in patients with OS $\leq 8$ months compared to $>8$ months OS (5/13 cases; $42.5 \%)$. Furthermore, the OS was 12 months in cases exhibited low complex karyotypes compared to 6.5 months in patients with high complex karyotypes (Table 1). These differences were statistically significant $(p<.05)$ suggesting chromosome complexity predicts shorter survival. Cases with loss of chromosome 13 or deletion 13q $(n=9,29 \%)$ exhibited significantly shorter OS (3 months), compared to cases with retained chromosome $13 q$ (OS $=9.5$ months, $p=.04$ ). Aberrations of $17 p$ (TP53 deletion by FISH, loss of $17,17 p$ deletion, or $17 p$ rearrangement by karyotype) were detected in a significant number of cases $(n=11$, $35.4 \%$ ), and showed a worse outcome (median OS $=6.5$ months for $17 p$ abnormalities versus 10.5 months for cases without $17 p$ abnormalities), although not statistically significant. Cases with p16 (CDKN2A) deletion $(n=2)$ and HSR's $(n=2)$ had short OS (10 days and 2 months, and 15 and 3 months, respectively). Discordant karyotypes were seen in two samples from one patient $(\# 15)$ obtained 4 years apart, with the later specimen exhibiting a complex karyotype with TP53 deletion. One case (\#10) showed karyotype progression within a few months, with acquisition of HSR. Both these events were associated with very short survival. These findings further suggest that deletions of $17 p$ (TP53) and 9p (CDKN2A), as well gene amplifications may serve as poor prognostic markers in ATLL. Aberrations of ploidy, clonal heterogeneity, treatment-naïve versus treated cases, acute versus lymphomatous subtypes did not show statistically significant differences in overall survival. In addition, we compared the observed cytogenetic abnormalities with two known prognostic indices in ATLL: serum calcium and lactate dehydrogenase (LDH) levels. We found patients with loss of chromosome 13 or deletion $13 q$ had significantly higher LDH levels (mean $=2571 \mathrm{mg} / \mathrm{dL}$ ) compared to patients without (mean $=1058 \mathrm{mg} / \mathrm{dL}, p<.05$ ). No cytogenetic aberration was found to be significantly correlated with calcium levels.

To the best of our knowledge, this is the largest series of cytogenetic analysis of the Caribbean ATLL patients in the United States. The presence of high complex chromosomal aberrations was a frequent feature of our Caribbean ATLL cohort and included numerical abnormalities as well as structural chromosomal rearrangements. In a recent study of Caribbean ATLL patients [5], which included 13 patients with abnormal cytogenetics (12 acute and 1 lymphomatous variants), the authors found the most commonly affected chromosome was $14(8 / 13$, $61.5 \%)$, followed by chromosomes 1, 9, 11, and $20(46.2 \%$ each). Recurrent deletions noted were deletions of 3q21, $5 q 33$, and $20 q 11.2$. Some of these findings are similar to our cohort, such as chromosome 14 being the most commonly affected chromosome and high percentage of recurrent deletion involving $3 q 21$. However, many of their findings are different from our study, probably due to low number of cases and higher percentage of acute variant of ATLL included in their cohort. No clinical correlations were reported in the study.

Although several of the cytogenetic features in our cohort were similar to Japanese ATLL, certain significant variations in frequency of specific chromosomal abnormalities were observed. In the largest Japanese study [8], the most frequent numerical changes in chromosomes were trisomy $3(21 \%), 7(10 \%)$, and $21(9 \%)$, monosomy $X$ (38\% in females), and $Y(17 \%$ in males), while our cohort showed high frequency of copy number loss in chromosome $14(29.0 \%)$, followed by 13 and 19 (22.6\% each), and 5 and 17 (19.4\% each). The most common recurrent chromosomal rearrangement breakpoints in the Japanese study was $14 q 32(28 \%)$, followed by $14 q 11(14 \%)$, while in our cohort were 6q21 (25.8\%), 1q21 (19.4\%), 3p21 (19.4\%), and $14 q 32$ (16.1\%). Similar to the Japanese studies $[9,11]$, deletions most frequently involved $6 q$ in our cohort (29.0\%). Our cohort also showed much higher frequency of cases with high complex karyotype ( $>10$ aberrations, 61.3\%) which was associated with significant 
shorter survival. Similarly, in the Japanese study [9], multiple chromosomal breaks $(\geq 6,42 \%)$ were found to correlate with shorter overall survival. In addition, they found abnormalities of many chromosomes (e.g. 1p, 1p22, 1q, 1q10-21, 2q, 3q, 3q10-12, 3q21, 14q, and 14q32) correlated with shorter survival, which we did not find in our cohort. Instead, we found loss of chromosome 13 or del13q to be associated with statistically significant shorter survival. Aberrations of $17 p$ were seen in a large proportion of our cases (35.5\%) and associated with shorter survival, which is in accord with published data from Japanese ATLL $[9,10]$.

Some factors might have contributed to the observed cytogenetic differences between Caribbean and Japanese ATLL patients. For example, our cohort included only aggressive clinical variants (lymphomatous and acute), while Japanese studies included also indolent variants (chronic and smoldering); Many of our patients had relapsed/refractory disease $(12 / 31,38.7 \%)$ when initially presented to our hospital, compared to newly diagnosed ATLL patients in the Japanese cohorts. Despite these confounding factors, the Caribbean ATLL is likely biologically distinct from the Japanese ATLL. Indeed, a most recent study reported targeted exon sequencing in 30 North American ATLL patients and compared the results to the Japanese ATLL cases [12]. The authors found a higher percentage of mutations in epigenetic and histone modifying genes and lower frequency of mutations in JAK/ STAT and TCR/NF- KB pathway genes in their North American ATLL cohort as compared to the Japanese ATLL. This is the first molecular genetic data on Caribbean ATLL patients and further supports the distinction between Caribbean and Japanese ATLL at the molecular level. Further studies with more Caribbean ATLL cases included are needed to consolidate these findings and to determine the role of host and/or environmental socioeconomic factors, or differences in clinical care and therapy.

Potential conflict of interest: Disclosure forms provided by the authors are available with the full text of this article online at https://doi.org/10.1080/10428194.2018.1538506.

\section{References}

[1] Hisada M, Stuver SO, Okayama A, et al. Persistent paradox of natural history of human $T$ lymphotropic virus type I: parallel analyses of Japanese and Jamaican carriers. J Infect Dis. 2004;190:1605-1609.

[2] Phillips AA, Shapira I, Willim RD, et al. A critical analysis of prognostic factors in North American patients with human Tcell lymphotropic virus type-1-associated adult T-cell leukemia/lymphoma: a multicenter clinicopathologic experience and new prognostic score. Cancer. 2010;116:3438-3446.

[3] Hanchard B. Adult T-cell leukemia/lymphoma in Jamaica: 1986-1995. J Acquir Immune Defic Syndr Hum Retrovirol. 1996; 13:S20-S25.

[4] Manns A, Cleghorn FR, Falk RT, et al. Role of HTLV-I in development of non-Hodgkin lymphoma in Jamaica and Trinidad and Tobago. The HTLV Lymphoma Study Group. Lancet. 1993;342:1447-1450.

[5] Zell M, Assal A, Derman O, et al. Adult T-cell leukemia/lymphoma in the Caribbean cohort is a distinct clinical entity with dismal response to conventional chemotherapy. Oncotarget. 2016;7:51981-51990.

[6] Zell Ml, Amer A, Bhavana K, et al. Analysis of large cohort shows that Caribbean adult $\mathrm{T}$ cell leukemia/lymphoma is a chemotherapy refractory disease with very poor prognosis that behaves distinctly from Japanese subtypes. Blood. 2014; 124:1685.

[7] Malpica L, Pimentel A, Reis IM, et al. Epidemiology, clinical features, and outcome of HTLV-1-related ATLL in an area of prevalence in the United States. Blood Adv. 2018;2:607-620.

[8] Kamada N, Sakurai M, Miyamoto K, et al. Chromosome abnormalities in adult T-cell leukemia/lymphoma: a karyotype review committee report. Cancer Res. 1992;52: 1481-1493.

[9] Itoyama T, Chaganti RS, Yamada Y, et al. Cytogenetic analysis and clinical significance in adult T-cell leukemia/lymphoma: a study of 50 cases from the human T-cell leukemia virus type1 endemic area, Nagasaki. Blood. 2001;97:3612-3620.

[10] Hatta $Y$, Yamada $Y$, Tomonaga $M$, et al. Allelotype analysis of adult T-cell leukemia. Blood. 1998;92:2113-2117.

[11] Tagawa H, Miura I, Suzuki R, et al. Molecular cytogenetic analysis of the breakpoint region at 6q21-22 in T-cell lymphoma/leukemia cell lines. Genes Chromosomes Cancer. 2002; 34:175-185.

[12] Shah UA, Chung EY, Giricz O, et al. North American ATLL has a distinct mutational and transcriptional profile and responds to epigenetic therapies. Blood. 2018;132(14):1507-1518. 